\title{
Fifty cases of late prosthetic valve endocarditis: improvement in prognosis over a 15 year period
}

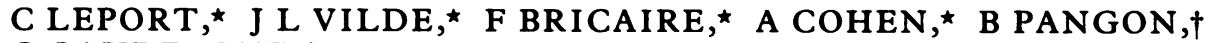 \\ C GAUDEBOUT $\ddagger$ P E VALERE $\S$
}

From the `Service des Maladies Infectieuses et Tropicales, $\nmid$ Laboratoire de Microbiologie, $\ddagger I N S E R M$ U13 Hôpital Claude Bernard; and §Service de Cardiologie, Hôpital Beaujon, Paris, France

SUMMARY The clinical course, prognostic factors, and management of 50 cases of late prosthetic valve endocarditis, occurring more than two months after valve replacement, were reviewed. Twenty nine cases that presented from 1971 to 1980 were compared with 21 cases that presented from 1981 to 1985. Apart from an appreciable decrease in the frequency of neurological complications between the first period $(38 \%)$ and the second period $(10 \%)$ no differences in clinical or bacteriological features were seen. Seventeen (59\%) of the 29 cases in the earlier period and four $(19 \%)$ of the 21 cases in the later period died. The rationale for antimicrobial treatment was similar during both periods. Cardiac surgery was performed in eight of 29 cases between 1971 and 1980 and in 11 of 21 between 1981 and 1985; the mean (SD) time between diagnosis of endocarditis and operation was 28 (19) days and 43 (44) days respectively. Six of the eight cases operated on in the first period died as did two of the 11 operated on in the second period. Twenty seven of the 29 cases presenting between 1971 and 1980 were treated with anticoagulants-either warfarin (15 of 27) or heparin sodium (12 of 27). Sixteen of the 21 cases presenting later were given anticoagulants and 15 of these cases were given heparin sodium. Control of anticoagulation was inadequate in nine of the 27 cases treated with anticoagulants during the first period and in only two of 16 treated during the second period. During the first treatment period neurological complications were more frequent when control of anticoagulation was inadequate.

Since the first reports of prosthetic valve endocarditis in $1962-63^{1}$ the number of cases has increased. This is the result of an increase in the number of patients undergoing valve replacement and of the longer survival of these patients. The overall prevalence of late prosthetic valve endocarditis, however, remains approximately $1.2 \%{ }^{2}$

In a review in 1979 of 140 cases of late prosthetic valve endocarditis Watanakunakorn reported a mean death rate of $45 \% .{ }^{3}$ The wide variation in mortality (from $18 \%$ to $67 \%$ ) in various series indicates that many factors can influence prognosis. ${ }^{45}$

We have already reported our experience with 29 cases of late prosthetic valve endocarditis studied retrospectively from 1971 to 1980 at the Claude Bernard Hospital. ${ }^{6}$ We have reviewed a further 21 cases

Requests for reprints to Dr C Leport, Service des Maladies Infectieuses et Tropicales, Hôpital Claude Bernard, 10 avenue de la Porte d'Aubervilliers, 75019 Paris, France.

Accepted for publication 20 January 1987 examined between 1981 and 1985 and we have compared them with the earlier series in an attempt to analyse the factors contributing to any changes in the pattern and prognosis of the disease.

\section{Patients and methods}

From January 1971 to December 1985 there were 50 cases of late prosthetic valve endocarditis among the 449 cases of infective endocarditis seen at the Department of Infectious and Tropical Diseases, Claude Bernard Hospital, Paris.

Late prosthetic valve endocarditis was defined as an infection occurring more than two months after valve replacement. ${ }^{7}$ Diagnosis was based on the following criteria: (a) anatomical evidence of endocarditis (22/50 cases) and (b) at least two positive blood cultures containing the same organism and a consistent clinical picture (43 of 50 cases). In the seven cases with negative blood cultures the diagnosis was confirmed anatomically in five and was prob- 


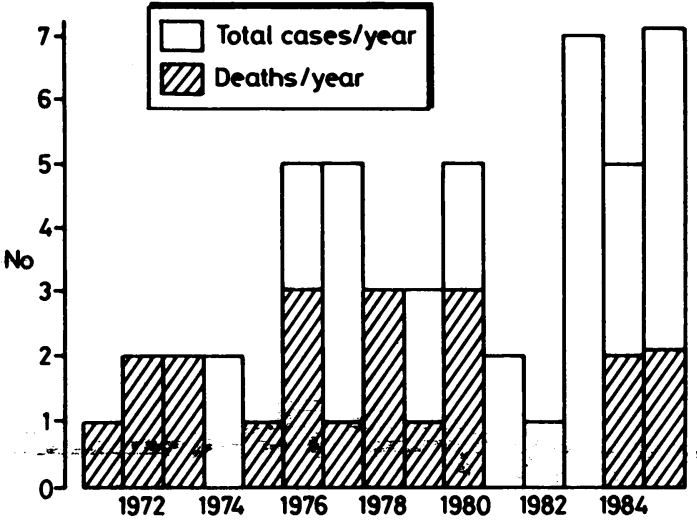

Figure Number of cases and mortality of late prosthetic valve endocarditis, 1971-85.

able in two. A relapse was defined as a subsequent episode of endocarditis in which the same organism as in the original episode was implicated and a recurrence as a subsequent episode in which an organism other than the original one was implicated.

The cases were divided into two groups (figure).Twenty nine cases occurred during the earlier period (between 1971 and 1980) in 26 patients (17 men and nine women; mean (SD) age 51 (14) years (range 20-70)). One patient had three recurrences. There were twenty one further cases during the later period (between 1981 and 1985) in 20 patients (13 men and seven women; mean (SD) age 46(19) years, range 11-75) and one patient had a recurrence.

The two periods were compared by the $\chi^{2}$ test (both with and without Yates' correction), the Fisher exact probability test, the Mann-Whitney U test, the analysis of variance, and.Student's $t$ test. A p value of $\leqslant 0.05$ was taken to indicate statistical significance.

\section{Results}

\section{CLINICAL AND BACTERIOLOGICAL FEATURES}

During the first period all 29 cases had mechanical valves, whereas during the second period eight of 21 had heterograft valves $(p<0.001)$. The sites of the prostheses were similar in the two periods (table 1 ).

The only significant difference in the clinical features of the disease between the two periods was the decrease in cerebral events, from $11(38 \%)$ of 29 cases in 1971-80 to two (10\%) of 21 cases in 1981-85 (p < 0.05) (table 2).

There was no significant difference in the bacteriological profile between the two groups (table 3 ). The frequency of non-streptococcal endocarditis (16/29 in 1971-80 and $11 / 21$ in 1981-85) was similar during the two periods.
Table 1 Comparison of the site of the prostheses in late prosthetic valve endocarditis between two groups treated over two periods

\begin{tabular}{lcc}
\hline & \multicolumn{2}{l}{ Number of cases } \\
\cline { 2 - 3 } & $1971-80$ & $1981-85$ \\
\hline Aortic & 9 & 12 \\
Mitral & 8 & 3 \\
Tricuspid & 0 & 3 \\
Multiple & 12 & 3 \\
Total & 29 & 21 \\
\hline
\end{tabular}

Table 2 Clinical features in cases of late prosthetic valve endocarditis during the troo periods

\begin{tabular}{llc}
\hline & \multicolumn{2}{l}{ Number of cases $(\%)$} \\
\cline { 2 - 3 } & $1971-80$ & $1981-85$ \\
& $(29$ cases) & $(21$ cases) \\
\hline Fever & $29(100)$ & $20(95)$ \\
Splenomegaly & $7(24)$ & $5(24)$ \\
New heart murmur & $13(45)$ & $11(52)$ \\
Congestive heart failure & $17(59)$ & $10(48)$ \\
Cerebral event & $11^{\star}(38)$ & $2 \star(10)$ \\
\hline
\end{tabular}

‡The only significant difference between the two periods was that for cerebral events $(p<0.05)$.

Table 3 Bacteriological features of cases of late prosthetic valve endocarditis during the two periods

\begin{tabular}{lcc}
\hline & \multicolumn{2}{c}{ Number of cases (\%) } \\
\cline { 2 - 3 } & $1971-80$ & $1981-85$ \\
\hline Streptococcus: & $13^{\star}(45)$ & $10^{\star}(48)$ \\
Str enterococcus (group D) & 7 & 4 \\
Str bovis (group D) & 3 & 1 \\
Non-group D & 3 & 5 \\
Staphylococcus: & $10(34)$ & $3(14)$ \\
Staph aureus & 6 & 2 \\
Coagulase negative & 4 & 0 \\
micrococcus sp & 0 & 1 \\
Other micro-organism & $2 \dagger$ & $3 \ddagger$ \\
Two causative agents & $3 \$$ & $1 \uparrow$ \\
Negative blood and valve cultures & 1 & $4 \star \star$ \\
Total & 29 & 21 \\
\hline
\end{tabular}

*Frequency of streptococcal endocarditis in the two periods $(\mathrm{p}=\mathrm{NS})$.

tKlebsiella in one case and Pseudomonas in the other.

†Corynebacterium in one case, Cardiobacterium hominis in one, and Histoplasma capsulatum in one case.

$\S$ Streptococcus faecalis and Pseudomonas stutzeri in one case, Staphylococcus coagulase negative and Candida tropicalis in one case, and Streptococcus faecalis and Enterobacter cloacae in one case. I Streptococcus sanguis 1 and Streptococcus sanguis 2 in one case.

$\star \star$ Serological evidence of Coxiella burnetii endocarditis in one case.

\section{OU TCOME}

The overall mortality for the two periods was $42 \%$ ( 21 of 50 cases). Seventeen (59\%) cases in the first group died and four $(19 \%)$ in the second $(p<0.01)$. Death was related to cardiac failure in 10 of the 17 earlier cases and in two of the four later cases; a neurological complication accounted for the deaths of 
seven of 17 cases from 1971 to 1980 and two of the four cases from 1981 to 1985.

The only relapse occurred in the more recent period in a patient who was treated with antimicrobials alone for endocarditis caused by Streptococcus $D$ faecium. This patient was successfully treated with antimicrobials and valve replacement. Between 1971 and 1980 one patient died after three recurrences caused by Streptococcus faecalis, and finally Streptococcus bovis and Pseudomonas aeruginosa. Between 1981 and 1985 there was one recurrence caused by Straphylococcus aureus in a drug addict with a tricuspid prosthesis who had previously been infected with Streptococcus sanguis.

\section{ANTIMICROBIAL TREATMENT}

All but one of the 50 cases was treated with antibiotics; in the first period one patient died before treatment could be started. The choice of antimicrobial agents was based on the same principles during both periods; the agent was selected according to the causative organism, the in vitro susceptibility tests, and the serum bactericidal titre. A combined drug regimen was used in 45 of 47 cases of non-fungal endocarditis. The mean (SD) interval between the first manifestations of endocarditis and the onset of antimicrobial treatment was $23(18)$ days (range 2-59) for 1971-80 and 25(36) days (range 2-145) for 1981-85 (NS). The duration of treatment in non-fungal endocarditis was significantly longer in recent years- 54 (32) days (range 3-146) versus 34 (20) days (range 2-80) ( $<<0.01$ ). In 1971-80, however, seven of 27 patients died before day 15 of treatment, whereas in 1981-85 only one of 20 patients did.

\section{SURGICAL TREATMENT}

Nineteen patients underwent valve replacement during the active phase of the disease (eight of the 29 initial cases and 11 of the 21 more recent cases). Analysis of prognostic factors from 1971 to $1980^{6}$ led to more precise indications for operation being applied in recent years; these were based on criteria established by Karchmer et al. ${ }^{8}$ Thus operation was more common during 1981-85; however, this difference was not statistically significant $(p=0.08)$. The mean (SD) interval between the diagnosis of endocarditis and the valve replacement was not significantly different during the two periods: 28 (19) days (range 2-60) in 1971-80 and 43(44) days (range 8-150) in 1981-85. In the first period, six $(75 \%)$ of eight surgically treated patients died and in the second period two $(18 \%)$ of 11 died $(p<$ 0.05 ). Culture of the prosthesis in 17 of 19 surgically treated cases was sterile in eight cases. In seven others it grew the same or one of the organism(s) present in the blood cultures and in two cases it grew an organism which had not been isolated from the blood-Histoplasma capsulatum in one case and Streptococcus mitis in the other.

\section{ANTICOAGULANT TREATMENT}

Anticoagulant treatment was continued in 43 of 50 cases in which it had been used before the development of endocarditis. From 1971 to 1980, 27 of 29 cases were given anticoagulants-15 warfarin and 12 heparin sodium. During this period two patients treated with warfarin had neuromeningeal bleeding with a prothrombin time of less than $10 \%$, without the dose of anticoagulant being changed. For this reason during the second period we treated 15 of 16 cases with heparin sodium rather than warfarin. Satisfactory coagulation tests (that is, activated partial thromboplastin time between 1.5 and 2.5 times control or prothrombin time between $20 \%$ and $40 \%$ control) were more often maintained during recent years, 14 of 16 cases, than in the past, 18 of 27 cases.

During the first period, neurological complications were more frequent when anticoagulation was inadequate $(p<0.05)$ (table 4). Between 1971 and 1985 two of the seven patients who were not treated with anticoagulants had a cerebral embolus and one had cerebral bleeding related to severe thrombopenia. Neurological signs were an early manifestation of endocarditis occurring before the start of treatment, in six of 11 cases between 1971 and 1980 and in the two recent cases with cerebral events.

\section{PROGNOSTIC FACTORS}

There was no significant difference in mortality (expressed as a percentage of cases) between men $(12$ of $30(40 \%))$ and women (nine of $16(56 \%)$ ), or between cases with mitral endocarditis (five of 11 $(45 \%))$, aortic endocarditis (eight of $21(38 \%)$ ), tri-

Table 4 Correlation between neurological complications and anticoagulant treatment in cases of late prosthetic valve endocarditis occurring during two periods

\begin{tabular}{|c|c|c|}
\hline & \multicolumn{2}{|c|}{$\begin{array}{l}\text { Number of cases with } \\
\text { cerebral events as a } \\
\text { proportion of cases }\end{array}$} \\
\hline & $1971-80$ & $1981-85$ \\
\hline $\begin{array}{l}\text { Anticoagulants given } \\
\text { Adequate anticoagulation } \\
\text { Inadequate anticoagulation } \\
\text { Anticoagulants not given } \\
\text { Total }\end{array}$ & $\begin{array}{l}9 / 27 \\
3+/ 18^{\star} \\
6 \ddagger / 9 \star \\
2 / 2 \\
11 / 29\end{array}$ & $\begin{array}{l}1 / 16 \\
1+/ 14 \\
0 / 2 \\
1 / 5 \\
2 / 21\end{array}$ \\
\hline
\end{tabular}


cuspid endocarditis (one of three $(33 \%)$ ), or multiple valve endocarditis (seven of $15(47 \%))$. The age of these who died and those who survived was similar. When heterograft valves were affected the death rate was $25 \%$ (two of eight cases). This was not significantly different from the death rate in endocarditis of mechanical valves (19 of 42 cases ( $45 \%$ )). The results of cross sectional echocardiography combined with pulsed Doppler could only be studied from 1981 to 1985 . This technique provided evidence for the diagnosis of endocarditis-that is, vegetations (seven cases) or valve dysfunction (six cases) in 11 of the 19 cases studied. Two of the seven cases with vegetations died. Six of them had had valve replacement.

Several factors were associated with a less favourable outcome (tables 5 and 6 ). The predictive value (that is the number of deaths compared with the

Table 5 Comparison of factors in the prognosis in late prosthetic valve endocarditis between two periods. Figures are numbers of deaths/numbers of cases

\begin{tabular}{lcll}
\hline & $1971-80$ & $1981-85$ & Significance \\
\hline Cardiac failure with new & & & \\
$\quad$ regurgitant murmur & $8 / 9$ & $1 / 7$ & $\mathrm{p}=0.01$ \\
Conduction abnormality & $3 / 3$ & $\frac{1}{2}$ & \\
Neurological event & $10 / 11$ & $2 / 2$ & $\mathrm{NS}$ \\
Persistent fever & $6 / 9$ & $3 / 11$ & $\mathrm{NS}$ \\
Streptococcal infection & $3 / 13$ & $1 / 10$ & $\mathrm{NS}$ \\
Non-streptococcal infection & $14 / 16$ & $3 / 11$ & $\mathrm{p}<0.05$ \\
Total & $17 / 29$ & $4 / 21$ & $\mathrm{p}<0.01$ \\
\hline
\end{tabular}

NS, not significant.

Table 6 Comparison of the mortality in late prosthetic valve endocarditis between two periods, according to the treatment. Figures are numbers of deaths/number of cases $(\%)$

\begin{tabular}{lccl}
\hline & $1971-80$ & $1981-85$ & p value \\
\hline $\begin{array}{l}\text { Antimicrobial agents } \\
\quad \text { alone }\end{array}$ & $11 / 21(52)$ & $2 / 10(20)$ & NS \\
$\begin{array}{lccl}\text { Antimicrobial agents } \\
\quad \text { operation }\end{array}$ & $6 / 8(75)$ & $2 / 11(18)$ & $<0.05$ \\
Total & $17 / 29(59)$ & $4 / 21(19)$ & $<0.01$ \\
\hline NS, not significant. & & &
\end{tabular}

Table 7 Comparison of the mortality in late prosthetic valve endocarditis between two groups treated during two periods, according to the type of micro-organism and the treatment. Figures are numbers of deaths/number of cases

\begin{tabular}{lll}
\hline & $\begin{array}{l}\text { Antimicrobials } \\
\text { alone }\end{array}$ & $\begin{array}{l}\text { Antimicrobials } \\
\text { and operation }\end{array}$ \\
\hline Streptococcal endocarditis: & & \\
$1971-80$ & $3 / 11$ & $0 / 2$ \\
$1981-85$ & $0 / 5$ & $1 / 5$ \\
Non-streptococcal endocarditis: & $8 / 10$ & $6 / 6^{\star}$ \\
$1971-80$ & $2 / 5$ & $1 / 6^{\star}$ \\
$1981-85$ & & \\
\hline
\end{tabular}

${ }^{\star} \mathrm{p}=0.015$. number of cases with the risk factor) of atrioventricular conduction abnormality, fever persisting for over 10 days despite adequate antibiotic treatment, and neurological complication was similar during both periods. Cardiac failure caused by valve dysfunction was indicative of poor prognosis in the first period only. An isolated new regurgitant murmur was not associated with a poor prognosis-two patients of the eight cases died. Neither was operated upon. The prognosis of cases of nonstreptococcal endocarditis (14 deaths out of 16 cases) was significantly worse than the prognosis of cases of streptococcal endocarditis (three deaths out of 13 cases) between 1971 and 1980 ( $p<0.001)$. However, from 1981 to 1985 the outcome of these two groups was not different; there were three deaths out of 11 cases of non-streptococcal endocarditis and one death out of 10 cases of streptococcal endocarditis.

The mortality associated with the type of organism and treatment during the two periods could not be compared because the numbers of cases were too small. The prognosis of surgically treated cases of non-streptococcal endocarditis seems to be better in the second period, however (table 7).

\section{Discussion}

This study indicates a considerable improvement in the prognosis of late prosthetic valve endocarditis between 1971-80 and 1981-85. The overall mortality was similar to that seen in a review of published series in 1982 in which the case-fatality rate for 193 cases was $46 \%{ }^{2}$ Many factors may have contributed to this trend; some are related to the general improvement in medical care and surgical procedures and are thus difficult to analyse. Thus the differences between two successive periods must be interpreted with care. But an improvement in the treatment of late prosthetic valve endocarditis has been noted by others too. 4910

A comparison of clinical features in our study showed that the pattern of the disease has not changed significantly, except for a decrease in the frequency of neurological events in recent years. Neurological complications are one of the most important causes of death in prosthetic valve endocarditis ${ }^{611}$ and they are usually associated with a high death rate. ${ }^{8} \mathrm{~A}$ reduction in their occurrence may have been a major factor accounting for the improvement in prognosis between 1981 and 1985. This trend may be related to a change in the management of anticoagulants in recent years. The use of anticoagulants in late prosthetic valve endocarditis has been controversial. ${ }^{811-14}$ Wilson et al emphasised that they should be temporarily discontinued only if a neurological event occurred. ${ }^{15}$ In 
our series anticoagulant treatment was continued in all patients to whom they had been given before the development of endocarditis. The position of patients who are not on anticoagulants when endocarditis is diagnosed is still being debated. ${ }^{1112}$ The numbers of such patients have increased with the use of heterograft valves, which do not require long term anticoagulation.

Our study confirms that anticoagulation must be maintained in a narrow therapeutic range to decrease the frequency and the severity of neurological complications. ${ }^{1617} \mathrm{We}$ believe that intravenous heparin sodium is the best anticoagulant for patients with prosthetic valves. Stable anticoagulation cannot be achieved with warfarin, which may be affected by other drugs and infection. ${ }^{18}$ The increased use of heparin sodium in our patients probably contributed to the decrease in neurological complications and thus to the improvement in prognosis during recent years. The only two neurological events that occurred between 1981 and 1985 were the first manifestation of endocarditis in both cases. We believe that heparin sodium should be used routinely when anticoagulants are indicated and as soon as the diagnosis of late prosthetic valve endocarditis is suspected.

Bacteriological factors may also have influenced the changing prognosis of late prosthetic valve endocarditis. Like others we found that streptococcal endocarditis was associated with a better outcome than other infections. ${ }^{215}$ In our study the predominance of streptococci and the less common occurrence of staphylococci (especially Staphylococcus aureus) may have reduced mortality in 1981-85; however, the prognosis of streptococcal endocarditis was not different in the two periods, whereas there was a pronounced improvement in the outcome of non-streptococcal endocarditis in recent years.

Advances in treatment in the past few years have improved the prognosis of patients with prosthetic valve endocarditis. ${ }^{19}$ The effect of better medical management is difficult to assess. The principles of antimicrobial treatment remained the same-that is, a combined drug regimen of bactericidal agents with bacteriological tests ${ }^{215}$-however, more potent antibiotics may have improved the prognosis of patients with non-streptococcal endocarditis. ${ }^{20}$

Differences in surgical treatment between the two periods are more obvious. An analysis of prognostic factors from 1971 to $1980^{6}$ led us to formulate more precise indications for operation in recent years; these were based on the same criteria as those established by Karchmer et al. ${ }^{8}$ The significant decrease in mortality of surgically treated cases between the two periods is probably another important factor in the improved prognosis in our series. Similar findings were reported by Mayer and Schoenbaum ${ }^{2}$ when they reviewed five series. ${ }^{71621-23} \mathrm{We}$ found that improved surgical management ${ }^{24-26}$ was of particular benefit in those with non-streptococcal endocarditis (table 7).

Our study confirmed the validity of most of the criteria for operation established by Karchmer et al ${ }^{8}$ and reviewed by others. ${ }^{1527}$ Unlike others we did not find that aortic and mechanical valves were associated with a worse prognosis. ${ }^{5927}$ The presence of heterograft valves in the patients seen between 1981 and 1985, however, may have been instrumental in reducing the death rate. Cardiac failure due to valve dysfunction did not cause a higher death rate in recent years; all seven cases were treated by surgery. Embolic manifestations, infection with a fungal agent, and relapse have also been proposed as indications for operation, ${ }^{81527}$ but these cannot be evaluated in our study. The prognostic value of echocardiographic abnormalities must be confirmed by more extensive studies. Multiple factorial analysis of a greater number of cases may provide more precise indications for operation. Operation is an important factor in the improvement of prognosis of late nonstreptococcal prosthetic valve endocarditis.

We thank Dr P Rajagopalan for discussions and $\mathrm{J}$ Alix for linguistic assistance.

\section{References}

1 Geraci JE, Dale AJD, McGoon DC. Bacterial endocarditis and endarteritis following cardiac operations. Wis Med J 1963;62:302-15.

2 Mayer KH, Schoenbaum SC. Evaluation and management of prosthetic valve endocarditis. Prog Cardiovasc Dis 1982;25:43-54.

3 Watanakunakorn C. Prosthetic valve infective endocarditis. Prog Cardiovasc Dis 1979;22:181-92.

4 Richardson JV, Karp RB, Kirklin JW, Dismukes WE. Treatment of infective endocarditis. A 10 years comparative analysis. Circulation 1978;58:589-97.

5 Masur H, Johnson WD. Prosthetic valve endocarditis. $J$ Thorac Cardiovasc Surg 1980;80:31-7.

6 Leport C, Vilde JL, Bricaire F, et al. Late prosthetic valve endocarditis: bacteriological findings and prognosis in 29 cases. Eur Heart J 1984;5:139-43.

7 Dismukes WE, Karchmer AW, Buckley MJ, Austen WG, Swartz MN. Prosthetic valve endocarditis. Analysis of 38 cases. Circulation 1973;48:365-77.

8 Karchmer AW, Dismukes WE, Buckley MJ, Austen WG. Late prosthetic valve endocarditis. Clinical features influencing therapy. $A m J$ Med 1978;64: 199-202.

9 Rossiter SG, Stinson EB, Oyer PE, et al. Prosthetic valve endocarditis. Comparison of heterograft tissue valves and mechanical valves. $J$ Thorac Cardiovasc Surg 1978;76:795-802.

10 Westaby S, Oakley C, Sapsford RN, Bentall HH. Sur- 
gical treatment of infective endocarditis with special reference to prosthetic valve endocarditis. $\mathrm{Br} \mathrm{Med} J$ 1983;287:320-3.

11 Carpenter JL, McAllister CK, and the US Army Collaborative Group. Anticoagulation in prosthetic valve endocarditis. South Med J 1983;76:1372-5.

12 Horstkotte D, Korfer R, Loogen F, Rosin H, Bircks W. Prosthetic valve endocarditis. Clinical findings and management. Eur Heart J 1984;5:117-22.

13 Wilson WR, Geraci JE, Danielson GK, et al. Anticoagulant therapy and central nervous system complications in patients with prosthetic valve endocarditis. Circulation 1978;57:1004-7.

14 Johnson WD. Prosthetic valve endocarditis. In: Kaye $\mathrm{D}$, ed. Infective endocarditis. Baltimore: University Park Press, 1976:129-42.

15 Wilson WR, Danielson GK, Giuliani ER, Geraci JE. Prosthetic valve endocarditis. Mayo Clin Proc 1982;57:155-61.

16 Garvey GJ, Neu HC. Infective endocarditis: an evolving disease. Medicine (Baltimore) 1978;57:105-26.

17 Pruit IT, Rubin RH, Karchmer AW. Neurological complications of bacterial endocarditis. Medicine (Baltimore) 1978;57:329-43.

18 Qureshi GD, Reinders TP, Somori GJ, Evans HJ. Warfarin resistance with nafcillin therapy. $A n n$ Intern Med 1984;100:527-9.

19 Ivert TSA, Dismukes WE, Cobbs CG, et al. Prosthetic valve endocarditis. Circulation 1984;69:223-32.

20 Karchmer AW, Archer GL, Dismukes WE. Rifampin treatment of prosthetic valve endocarditis due to Staphylococcus epidermidis. Rev Infect Dis 1983;5: 543-8.

21 Slaughter L, Morris JF, Starr A. Prosthetic valvular endocarditis. A 12 year review. Circulation 1973;47: 1319-26.

22 Wilson WR, Jaumin PM, Danielson GK, et al. Prosthetic valve endocarditis. Ann Intern Med 1975;82: 751-6.

23 Saffle JR, Gardner P, Schoenbaum SC, Wild W. Prosthetic valve endocarditis. The case for prompt valve replacement. J Thorac Cardiovasc Surg 1977; 73:416-20.

24 Lau JKH, Robles A, Cherian A, Ross DN. Surgical treatment of prosthetic endocarditis. Aortic root replacement using a homograft. J Thorac Cardiovasc Surg 1984;87:712-6.

25 Donaldson RM, Ross DN. Homograft aortic root replacement for complicated prosthetic valve endocarditis. Circulation 1984;70:178-81.

26 Raychaudhury T, Cameron EWJ, Walbaum PR. Surgical management of prosthetic valve endocarditis. $J$ Thorac Cardiovasc Surg 1983;86:112-4.

27 Baumgartner WA, Miller C, Reitz BA, et al. Surgical treatment of prosthetic valve endocarditis. Ann Thorac Surg 1983;35:87-104. 